\title{
A Micro-Grid Battery Storage Management
}

\author{
Pukar Mahat, Member, IEEE, Jorge Escribano Jiménez, Eloy Rodriguez Moldes, Sandra Iren Haug, \\ Ireneusz Grzegorz Szczesny, Karl Eide Pollestad, Luminita Cristiana Totu
}

\begin{abstract}
An increase in number of distributed generation (DG) units in power system allows the possibility of setting-up and operating micro-grids. In addition to a number of technical advantages, micro-grid operation can also reduce running costs by optimally scheduling the generation and/or storage systems under its administration. This paper presents an optimized scheduling of a micro-grid battery storage system that takes into account the next-day forecasted load and generation profiles and spot electricity prices. Simulation results show that the battery system can be scheduled close to optimal even with forecast errors. ICLOCS software has been used for the numerical implementation.
\end{abstract}

Index Terms-Battery Storage, Distributed Generation, Forecasting, ICLOCS, Micro-grid, Optimization, Spot Price

\section{INTRODUCTION}

$\mathrm{F}$ OR a number of years, motivated by economics, decentralization policies and sustainability concerns, the trend in the power industry worldwide is to reduce investment and operation costs and to become environmentally friendly. This has resulted in an increased share of renewable energy (such as wind and solar) in the total generation capacity, and an increased number of small and medium sized geographically distributed power plants (such as wind turbines and district combined heat and power plants). The Danish power system is a forerunner example. This topological change in the power system landscape is opening up possibilities to form micro-grids: localized groups of DGs, storages and loads that act as autonomous power systems with a single point of common coupling to the main electricity network. Micro-grids meet the power quality and reliability requirements of the local customers [1], and also shield itself from issues such as voltage distortion, voltage sag, flicker, and lightning transients [2]. Apart from the technical benefits, micro-grids have also economic advantages [3] if appropriate control and management systems are implemented.

In [4], an agent based micro-grid management scheme is

P. Mahat, E. R. Moldes, S. I. Haug, I. G. Szczesny, K. E. Pollestad are with the Department of Energy Technology, Aalborg University, Pontoppidanstræde 101, Aalborg, DK-9220, Denmark (e-mails: pma@et.aau.dk, emolde11@student.aau.dk, shaug11@student.aau.dk, iszcze11@student.aau.dk and kpolle11@student.aau.dk).

J. Escribano Jiménez is with the Department of Mechanical Engineering, University of Zaragoza, María Luna 7, Campus Río Ebro, Edificio "Agustín de Betancourt", Zaragoza, 50018, Spain (e-mail jescribs@gmail.com)

L. C. Totu is with Department of Electronic Systems, Aalborg University, Fredrik Bajers Vej 7, Aalborg, DK-9220, Denmark (email: 1ct@es.aau.dk) proposed that only focus on balancing load and generation. A token based approach is presented in [5] for loss reduction and voltage support. A day-ahead power scheduling for the conventional and PV generators for micro-grid management is presented in [6]. A micro-grid management system to minimize fuel consumption by adjusting generators droop is presented in [7]. Another microgrid management strategies for multiple DG units to minimize the power import from the grid is presented in [8]. In [9], Bacterial Foraging Algorithm has been used to minimizing the cost function of the overall system while meeting the customer demand and safety of the system. It tries to find the best combination of micro turbine and fuel cell with change in load. This requires real time information on load, fast communication, fast algorithms and fast generations. An energy management scheme to share the power flow among the energy storages units is presented in [10]. In [11], Integer Programming optimization has been used to solve the optimal scheduling problem of the micro-grid. A fuzzy control is proposed for storage unit in micro-grid to maximize the profit of the micro-grid in [12]. Ant colony optimization (ACO) method for micro-grid dispatch control is presented in [13]. This multi-objective micro-grid management does not consider the load and generation forecast into consideration.

The present work proposes an optimal scheduling of the battery storage system in a manner similar to [14] and [15] but in addition takes into account the load and generation forecasts together with day-ahead electricity spot prices.

\section{MICRO-GRID MANAGEMENT}

In Denmark, the hourly spot prices of the electricity are settled a day-ahead in the Nord Pool market. In this paper, it is assumed that the electricity tariffs for small and medium consumers and producers such as micro-grids are based on the spot prices, although they do not participate directly in the market system.

The micro-grid management schedules the battery for the day-ahead by taking into account the hourly electricity tariffs and the load and generation forecasts. The objective is to minimize the micro-grid operation cost or equivalently maximize the profit, and the decision variables are the charging and discharging power of the battery storage system for each time interval of the day.

\section{A. Load and generation forecast}

The load and the generations, like wind generation, are stochastic in nature. It is important to forecast them so as to schedule the energy storage unit optimally. A load and wind 
generation data set covering a period of 3 months, with 15 minute time resolution, is used when making the day-ahead forecasts.

Various methods can be used to predict the wind speed or wind power [16]. In this work, the forecast uses only the past wind power generation data set. A non-differenced and nontransformed ARMA model is identified. The ARMA $(2,0)$ structure gave the best accuracy among a number of tested alternatives. Because of the large variability of wind generation in general, but also because of the highly simplified modeling and no weather forecast, the prediction error is as high as $38.41 \%$. However, the method is a good balance between complexity and performance.

The load is forecasted similar to the wind power generation, based only on the past load profiles data set. Among a number of tested alternatives, the ARMA $(1,1)$ model was found best fit with a prediction error of maximum $5.85 \%$. The low prediction error is in great part due to the highly periodic nature of the consumption profile from day to day. Generation and load forecast for the micro-gird for the day of simulation is shown in Fig. 1 and Fig. 2, respectively.

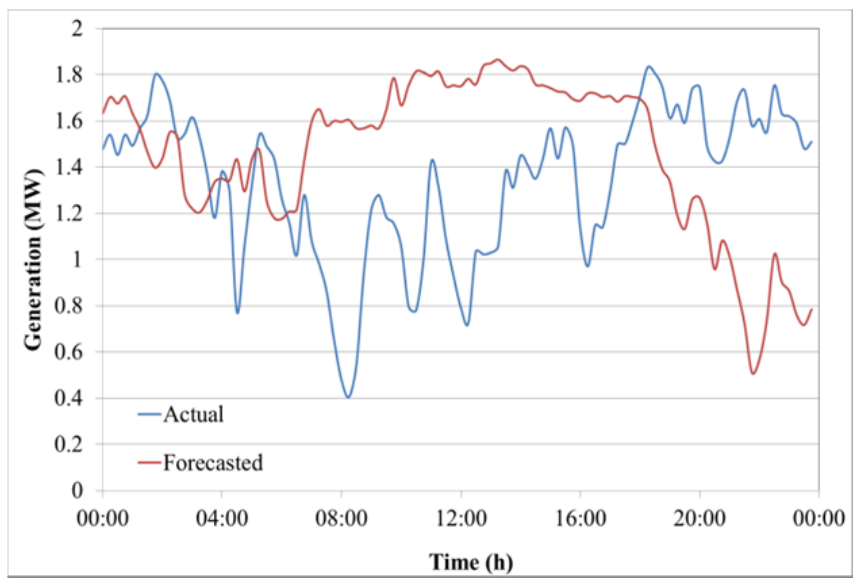

Fig. 1. Generation forecast

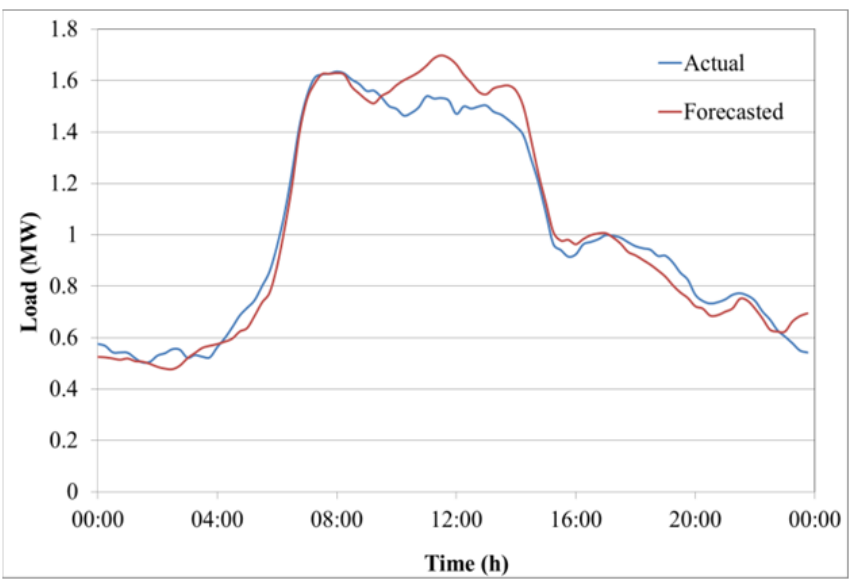

Fig. 2. Load forecast

\section{B. Optimization of the battery storage daily operation}

Considering a time horizon of $\mathrm{N}$ time samples, the objective of the battery storage optimization can be defined as

$$
\min _{u(\cdot)} \sum_{k=1}^{N} E_{\text {grid }}(k) C(k),
$$

Where $k$ is the time step index, $C(k)$ is the energy price in units of $[€ / \mathrm{kWh}]$ for a time period with index $k$, assumed to be equal for both buying and selling, and $E_{\text {grid }}(k)$ is the energy exchanged with the external power grid with positive values for energy import and negative values for energy export from the micro-grid. Furthermore, $E_{\text {grid }}(k)$ can be decomposed in the contributions of the forecasted local generation, forecasted local load, and the battery charging/discharging energy for the time period $k$ :

$$
E_{\text {grid }}(k)=E_{\text {gen }}(k)-E_{\text {load }}(k)+u(k) .
$$

The decision variables are the energy contributions of the battery, $u(k), k \in\{1,2, \ldots N\}$.

A time integrator model with no losses is used for the battery storage. There are constraints on the minimum and maximum energy level stored in the battery, and for the charge and discharge rates. This is written as:

$E_{\text {batt }}(k+1)=E_{\text {batt }}(k)+u(k)$,

$E_{\text {safe }}<E(k)<E_{\text {max }}$,

$|R| \times T_{s} \geq|u(k)|$,

where $T_{s}$ is the sample time, $E_{\text {batt }}(k)$ is energy stored in the battery at the beginning of time step $\mathrm{k}, u(k)$ is the scheduled charged $(+)$ or discharged (-) energy of the battery in a time sample, and $R$ is the maximum rate for both charging $(+)$ and discharging (-).

The above equations, written for each step $k$ of the time horizon, represent constraints for the optimization problem. It can be seen that both the objective and the constraints are linear in the decision variables, and the optimization of the battery storage system has thus been expressed as a linear optimization or linear programming.

\section{Implementation of the numerical optimization}

As discussed in the previous section, to find the battery schedule in the current formulation, a linear optimization problem needs to be solved. Linear programming is a wellstudied and both efficient algorithms and established software packages exist.

In this work, numerical simulations are carried out in the MATLAB-based optimal control software package ICLOCS [18]. ICLCOS can handle problems that are significantly broader in scope than the current linear formulation, making it possible to easily extend the dynamic model of the battery, the constraints, and the forecast models in the numerical simulation as future work.

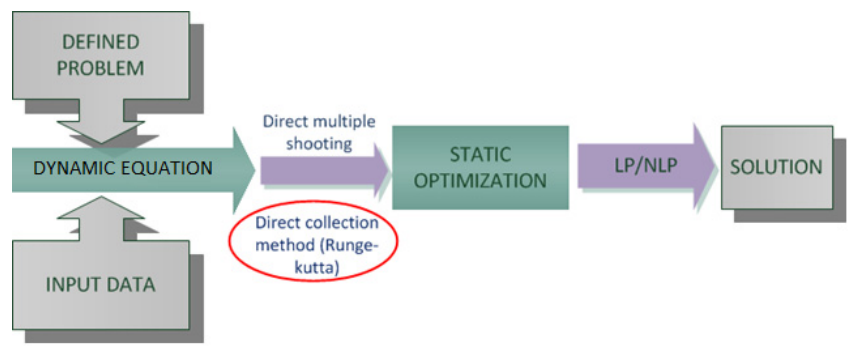

Fig. 3. Block diagram of ICLOCS 
One of the main advantages of using ICLOCS is that it takes care of transcribing the user-defined optimal control problem into a static optimization. The dynamical model in the problem, in this case the battery model, can be given in either the form of ordinary differential equations (continuous time) or difference equations (discrete time) and the associated equality constraints are generated automatically. To solve the static optimization problem, ICLOCS interfaces to a MATLAB built-in solvers or externally installed packages, linear, non-linear, convex or non-convex. This process flow is shown in Fig. 3.

\section{TEST MICRO-GRID}

A shopping center in Aalborg, Denmark is considered for the micro-grid setup. The data set consists of real consumption measurements and the power generation data from a $2 \mathrm{MW}$ near-by wind turbine. A $2 \mathrm{MWh}$ battery is added for this study. This will allow the micro-grid to run in island mode, for at least an hour without any wind power.

The safe level of the battery is $400 \mathrm{kWh}$ and the charging/discharging rate is $1600 \mathrm{~kW}$. The time interval is 15 minutes and, hence, there are 96 time intervals in the day. The state of charge of battery at the beginning and the end of the simulation period is $400 \mathrm{kWh}$.

The hourly electricity spot price, for the simulation day, is shown in Fig. 4. The historical data on load and generation is presented in Fig. 5.

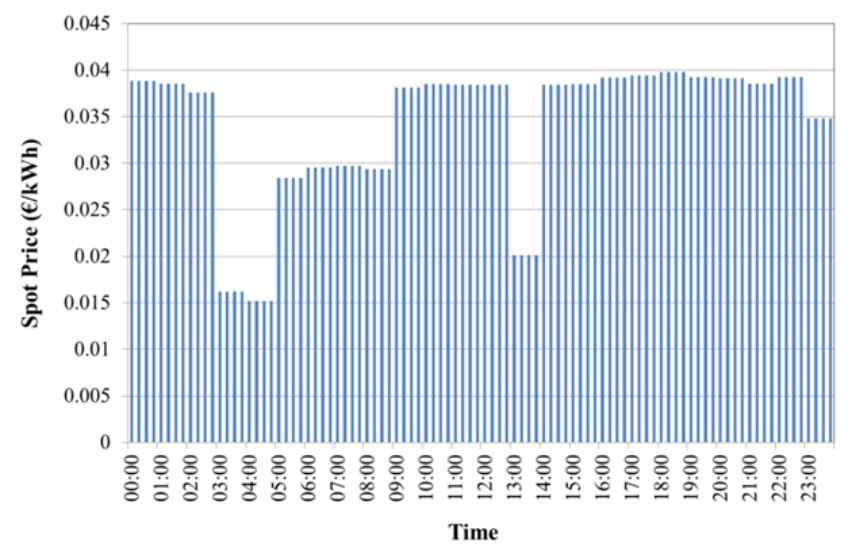

Fig. 4. Spot price of the electricity

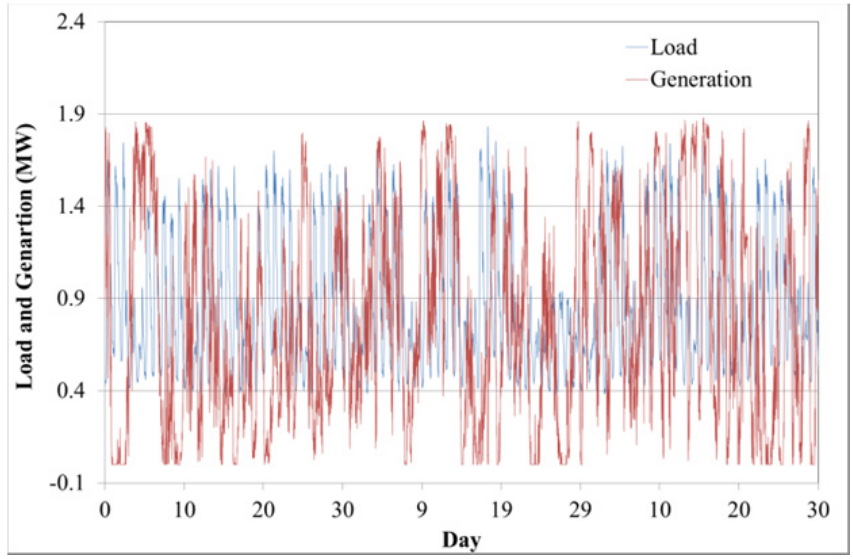

Fig. 5. Load and generation of micro-grid for 3 months

\section{RESUlTS AND Discussions}

The simulation has been conducted for three different cases. The first case uses the real measured data for the day. It corresponds to a case with no forecast errors, and it is used as benchmark reference for evaluating the other cases, as it produces the actual minimal cost for operation of micro-grid. The second optimization uses forecasted generation and load data and this gives the schedule of the battery and the forecasted operation cost of the micro-grid. The third case is the actual case, where the charge/discharge-cycle is based on the results of the optimization using the forecasted data, but the final settlement in price is based on the actual power exchange.

\section{A. Case 1}

The optimal scheduling of the battery is based on the actual wind generation and load consumption data of the simulation day. The optimal use of battery is obtained for every 15 minutes period and it is shown in Fig. 6. Positive values correspond to battery charging periods and negative values refer to battery discharging periods. The battery's state of the charge is shown in Fig. 7.

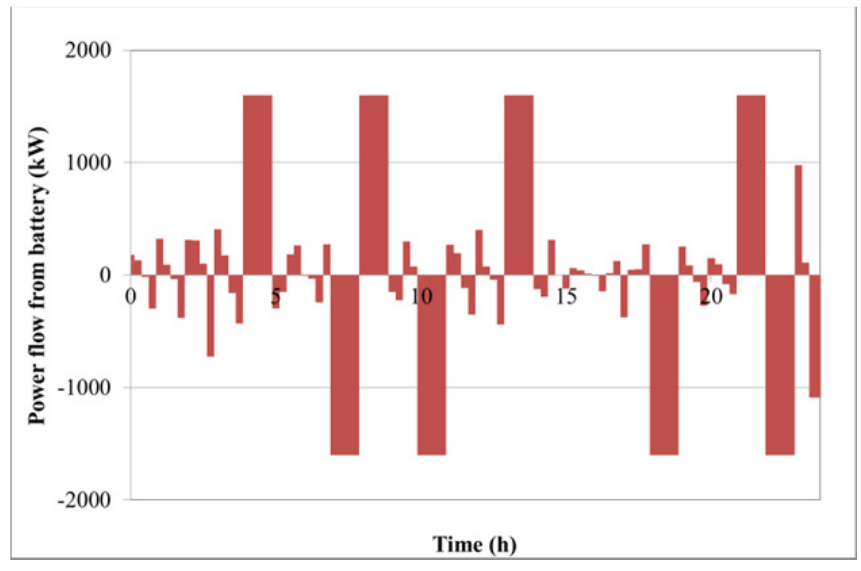

Fig. 6. Optimal use of batteries in Case 1

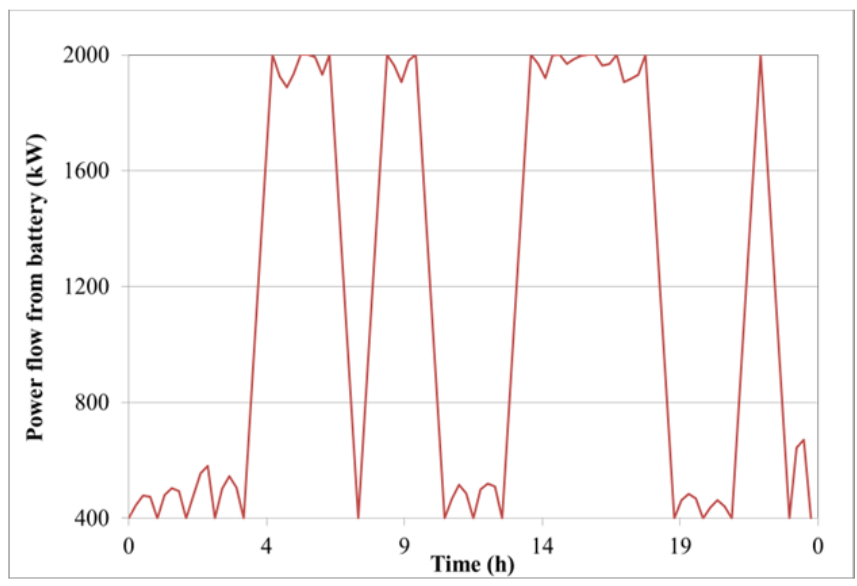

Fig. 7. Battery state of charge in Case 1

Fig . 8 shows the electricity exchange between the microgrid and the utility. A positive value indicates that the microgrid imports power while a negative value indicates export of 
power to the utility grid. Without battery, Fig. $8 \mathrm{a}$, the microgrid is exporting power almost all the time and the profit is $€ 361.38$. With the battery, Fig. 8b, power is imported during low price and sold during high price. The profit is increased to $€ 441.28$.

It would be ideal if all the loads and generations are known in advance and the micro-grid can be run optimally. However, generally they are not known in the advance. Thus, the battery must be scheduled based on the forecasted data as discussed in Case 2.

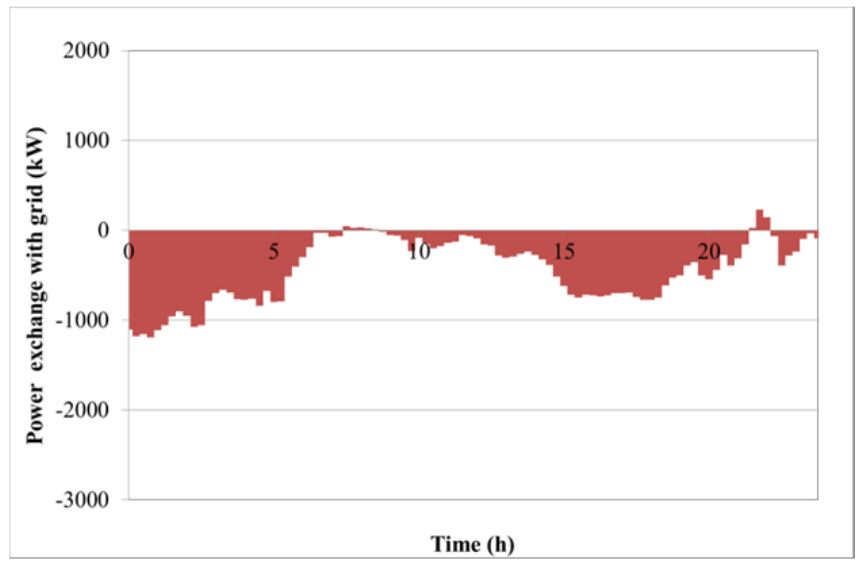

(a)

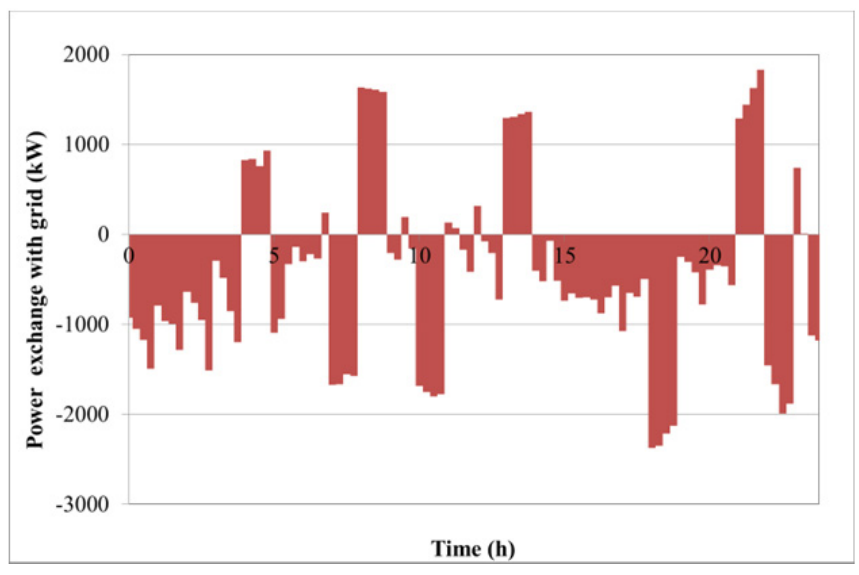

(b)

Fig . 8. Power exchange between micro-grid and grid in case 1 (a) without battery and (b) with battery

\section{B. Case 2}

In this case, the optimization is based on the predicted wind power generation and predicted load consumption. The optimal use of battery is shown in Fig . 9.

The state of charge of the battery is shown in Fig. 10. Similarly, the forecasted power exchange with the grid is shown in Fig. 11. The forecasted income of the micro-grid is $€ 358.96$. However, the actual settlement is based actual power production and consumption and battery uses. This is discussed in Case 3.

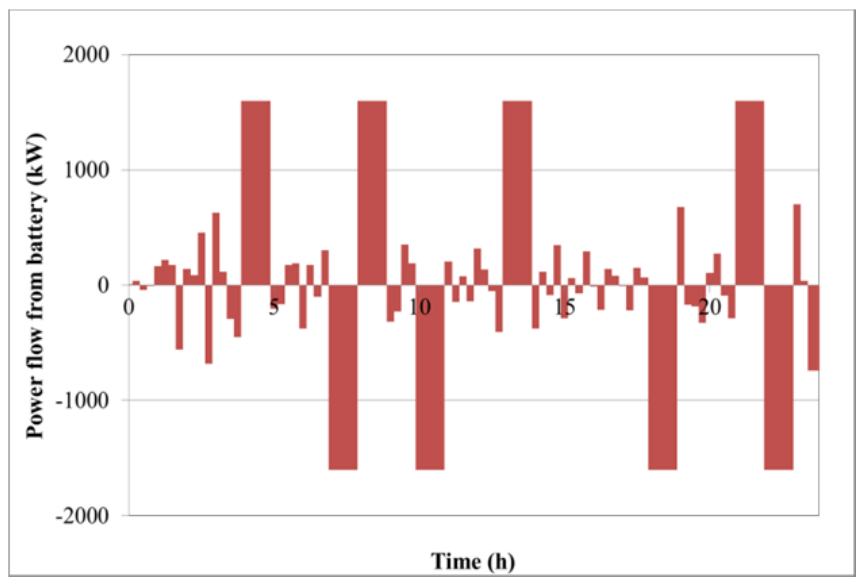

Fig. 9. Optimal use of batteries in Case 2

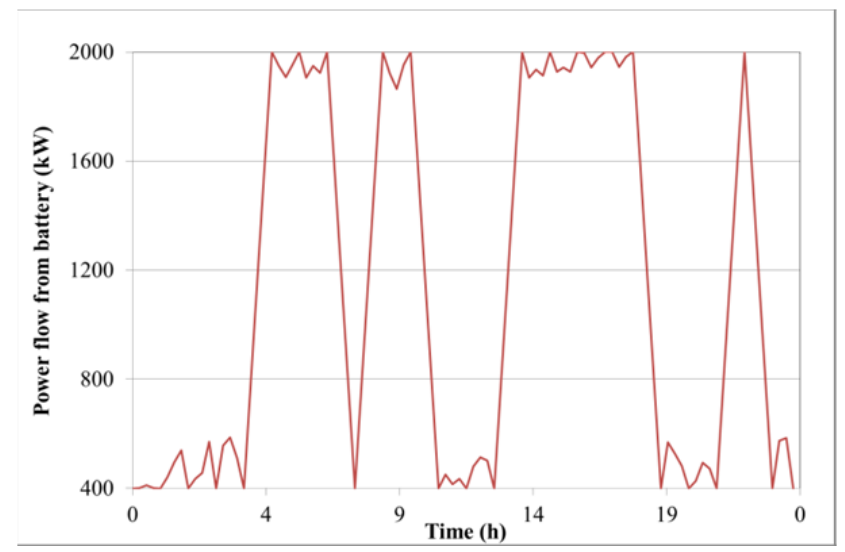

Fig. 10. Battery state of charge in Case 2

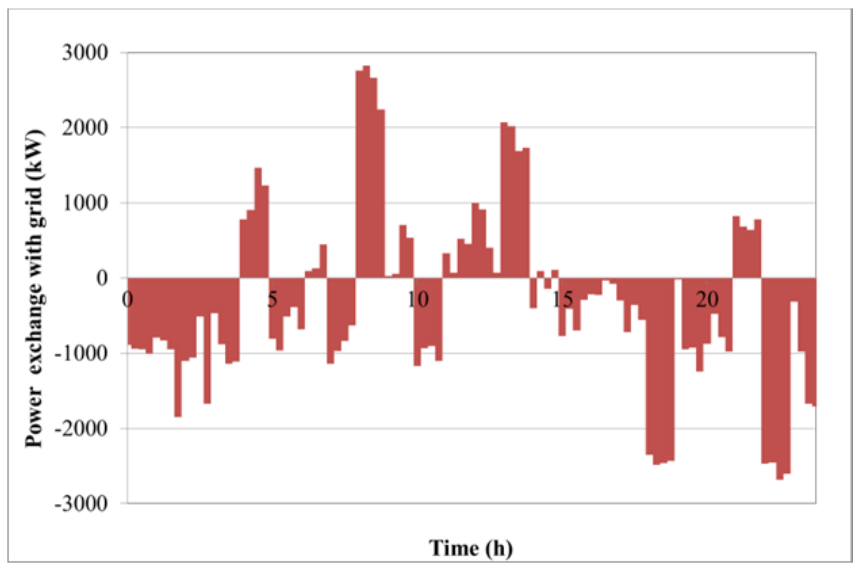

Fig. 11. Forecasted power exchange between micro-grid and main grid in Case 2

C. Case 3

In this scenario, battery is running according to the optimized schedule obtained at Case 2 and shown Fig. 9, which is based on the forecasted load and generation. But in Case 3, the load and generation are the actual ones, see also Fig. 2. In these conditions, the power exchange with the grid is shown in Fig. 12 and the income of the micro-grid is $€ 438.24$. 


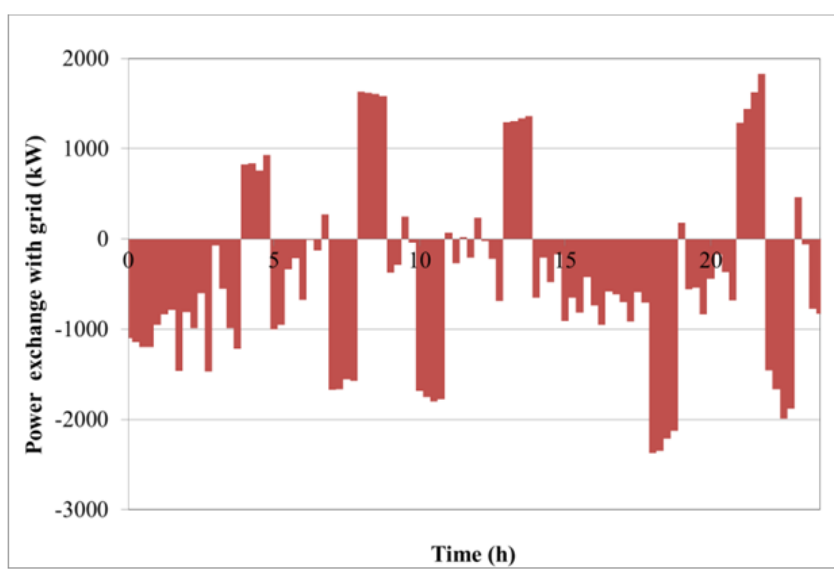

Fig. 12. Power exchange between micro-grid and main grid in Case 3

There is only a small difference in this final result compared to Case 1. Even with errors in the forecasting, the algorithm has obtained a near optimal. Also, it can be seen from Fig. 8, Fig. 11 and Fig. 12, that the micro-grid imports power from the main grid during the low price period even though the generation has covered the load demand. This is as expected as the extra energy is used to charge the battery making it possible to sell during high price hours. Thus the micro-grid makes more profit by optimizing the battery schedule using forecasts.

\section{CONCLUSIONS}

As the power system strives to become more efficient and environment friendly, the number of distributed generation, especially renewable energy based, is increasing thus resulting in the possibility of operating as micro-grids. Micro-grids can shield the local loads from various problems in the main grid by operating in island mode. However, this requires storage units when the DGs are based on intermitted sources, such as wind. While connected to the grid, the micro-grid can maximize its revenue or minimize its operating cost by optimally scheduling the battery. An optimization method and numerical simulations using the ICLOCS software have been presented in this paper. The load and generation forecast are taken into account to find the optimal schedule of the battery. The battery imports power during the cheap hours and exports it during expensive hours. Results show that micro-grid profits can be increased by optimally scheduling the battery. The method can be further improved by including the cost of the battery charging cycles as batteries have limited charging cycles.

\section{REFERENCES}

[1] C. Marnay, F. Rubio, and A. Siddiqui, "Shape of the microgrid," in IEEE PES Winter Meeting, Columbus, Ohio, USA, Jan. 2001, pp. 150153.

[2] IEEE guide for design, operation, and integration of distributed resource island systems with electric power systems, IEEE Standard 1547TM, July 2011.

[3] Z. Zhang, G. Li, and M. Zhou, "Application of microgrid in distributed generation together with the benefit research," in IEEE PES General Meeting, Minnesota, USA, Jul. 2010, pp. 1-5.

[4] J. Oyarzabal, J. Jimeno, J. Ruela, A. Engler and C. Hardt, “Agent based micro grid management system," in International Conference on Future Power Systems, Amsterdam, Netherlands, Nov. 2005, pp. 1-6.

[5] A. Costabeber, T. Erseghe, P. Tenti, S. Tomasin, and P. Mattavelli, "Optimization of micro-grid operation by dynamic grid mapping and token ring control," in 14th European Conference on Power Electronics and Applications, Birmingham, UK, Aug./Sep. 2011, pp. 1-10.

[6] R. Noroozian and H. Vahedi, "Optimal management of MicroGrid using Bacterial Foraging Algorithm," in 18th Iranian Conference on Electrical Engineering, Iran, May 2010, pp. 895-900.

[7] H. Kanchev, D. Lu, F. Colas, V. Lazarov, B. Francois, and S. Member, "Energy management and operational planning of a microgrid with a pvbased active generator for smart grid applications," IEEE Transactions on Industrial Electronics, vol. 58, no. 10, pp. 4583-4592, Oct. 2011.

[8] F. Katiraei and M. R. Iravani, "Power management strategies for a microgrid with multiple distributed generation units," IEEE Transactions on Power Systems, vol. 21, no. 4, pp. 1821-1831, Nov. 2006.

[9] E. Barklund, N. Pogaku, M. Prodanovic, T. C. Green, and S. Member, "Energy management in autonomous microgrid using stabilityconstrained droop control of inverters," IEEE Transactions On Power Electronics, vol. 23, no. 5, pp. 2346-2352, Sep. 2008.

[10] H. Zhou and T. Bhattacharya, "Composite energy storage system with flexible energy management capability for micro-grid applications," in IEEE Energy Conversion Congress and Exposition, Atlanta, Georgia, USA, Sep. 2010, pp. 2558-2563.

[11] S. Choi, et. al., "A microgrid energy management system for inducing optimal demand response," in IEEE International Conference on Smart Grid Communications, Brussels, Belgium, Oct. 2011, pp. 19-24.

[12] Y. Manjili, A. Rajaee, M. Jamshidi, and B. Kelly, "Fuzzy control of electricity storage unit for energy management of micro-grids," in World Automation Congress, Puerto Vallarta, Mexico, June 2012, pp. 1-6.

[13] C. Colson, M. Nehrir, and C. Wang, "Ant colony optimization for microgrid multi-objective power management," in IEEE PES Power Systems Conference and Exposition, Seattle, WA, USA, Mar. 2009, pp. $1-7$.

[14] A. Parisio and L. Glielmo, "Energy efficient microgrid management using Model Predictive Control," in IEEE Conference on Decision and Control and European Control Conference, Orlando, FL, USA, 2011, pp. 5449-5454.

[15] A. Bagherian and S. M. M. Tafreshi, "A developed energy management system for a microgrid in the competitive electricity market," IEEE Bucharest PowerTech, Bucharest, Romania, June/July 2009, pp. 1-6.

[16] S. Soman and H. Zareipour, "A review of wind power and wind speed forecasting methods with different time horizons," in North American Power Symposium, Arlington, TX, USA, Sep.2010, pp. 1-8.

[17] The Danish Energy Saving Trust, "Understanding your electricity bill," [Online]. Available:

http://www.savingtrust.dk/consumer/understandyour-energyconsumption/understanding-your-electricity-bill

[18] Imperial College London. Imperial College London Optimal Control Software User Guide (ICLOCS) [program]. Version 0.2. Manual Available: http://www.ee.ic.ac.uk/ICLOCS/user_guide.pdf 